\title{
Correction to: Electronic Voting
}

\author{
Robert Krimmer(D), Melanie Volkamer (D), Bernhard Beckert (D), \\ Ralf Küsters, Oksana Kulyk, David Duenas-Cid (D), \\ and Mihkel Solvak
}

\section{Correction to: \\ R. Krimmer et al. (Eds.): Electronic Voting, LNCS 12455, https://doi.org/10.1007/978-3-030-60347-2}

The original version of the cover and book was revised. The seventh editor name has been updated.

The original version of this chapter "A Unified Evaluation of Two-Candidate BallotPolling Election Auditing Methods" contains the following errors which have been now corrected:

Page 117:

- "It can be continuous, discrete, or neither." should read "It can be continuous, discrete, or a combination of the two."

Page 118:

- “...is risk-maximizing: for such a prior, limiting the upset probability to $\alpha$ also limits the risk to $\alpha$." should read "...is risk-maximizing. For such a prior, limiting the upset probability to $v$ also limits the risk: for the specific type of Bayesian audits considered by Vora [11], the risk limit is v; however, for the Bayesian audits described here (see below), the risk limit is $\frac{v}{1-v}>v . "$

- "The upset probability, $\operatorname{Pr}\left(H_{0} \mid Y_{n}\right)$, is not the risk, which we write informally as $\max _{H_{0}} \operatorname{Pr}\left(\right.$ certify $\left.\mid \mathrm{H}_{0}\right)$." should read " The upset probability, $\operatorname{Pr}\left(H_{0} \mid Y_{n}\right)$, is not the risk, which is $\operatorname{Pr}\left(\right.$ certify $\left.\| \mathrm{p}_{\mathrm{T}}\right)$."

- "For risk-maximizing priors, taking $h=\frac{1-\alpha}{\alpha}$ yields an audit with risk limit $\alpha . "$ should read "For risk-maximizing priors, taking $h=\frac{1}{\alpha}$ (which is equivalent to a threshold of $v=\frac{\alpha}{1+\alpha}$ on the upset probability) yields an audit with risk limit $\alpha . "$

Page 119:

- " $\operatorname{Pr}\left(Y_{n} \mid p_{1}\right)$ " should read " $\operatorname{Pr}\left(Y_{n} \| p_{1}\right) "$ (3 instances).

- " $\operatorname{Pr}\left(Y_{n} \mid p_{0}\right)$ " should read " $\operatorname{Pr}\left(Y_{n} \| p_{0}\right)$ " (1 instances).

The updated version of the book can be found at

https://doi.org/10.1007/978-3-030-60347-2

https://doi.org/10.1007/978-3-030-60347-2_8

(C) Springer Nature Switzerland AG 2021

R. Krimmer et al. (Eds.): E-Vote-ID 2020, LNCS 12455, p. C1, 2021.

https://doi.org/10.1007/978-3-030-60347-2_15 